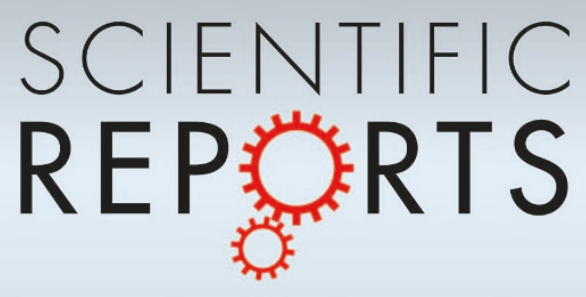

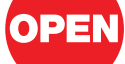

SUBJECT AREAS:

SUPER-RESOLUTION

MICROSCOPY

WIDE-FIELD FLUORESCENCE MICROSCOPY

BIOPHOTONICS

SINGLE-MOLECULE BIOPHYSICS

Received

6 December 2012

Accepted

4 January 2013

Published

4 February 2013

Correspondence and requests for materials should be addressed to H.I. (hyotcherl.ihee@ kaist.ac.kr); W.D.H. (wondo@kaist.ac.kr) or Y.K.P. lyk.park@ kasit.ac.kr)

* These authors contributed equally to this work.

$\uparrow$ Current address: Institute for Soft and Bio

Matter Science,

Changwon National University, Changwon, Kyungnam 641-773,

Republic of Korea.

\section{Simple super-resolution live-cell imaging based on diffusion-assisted Förster} resonance energy transfer

\author{
Sangyeon Cho ${ }^{*}$, Jaeduck Jang ${ }^{2 *}$, Chaeyeon Song ${ }^{2,3}$, Heeyoung Lee ${ }^{4}$, Prabhakar Ganesan ${ }^{1,5}$, \\ Tae-Young Yoon ${ }^{2}$, Mahn Won Kim ${ }^{2 \dagger}$, Myung Chul Choi ${ }^{3}$, Hyotcherl Ihee ${ }^{1,5}$, Won Do Heo ${ }^{4}$ \\ \& YongKeun Park ${ }^{2}$
}

\begin{abstract}
'Department of Chemistry, ${ }^{2}$ Department of Physics, ${ }^{3}$ Department of Bio and Brain Enginnering, ${ }^{4}$ Department of Biological Sciences, Korea Advanced Institute of Science and Technology, Daejeon 305-701, Republic of Korea, ${ }^{5}$ Center for Nanomaterials and Chemical Reactions, Institute for Basic Science, Daejeon 305-701, Republic of Korea.
\end{abstract}

\begin{abstract}
Despite the recent development of several super-resolution fluorescence microscopic techniques, there are still few techniques that can be readily employed in conventional imaging systems. We present a very simple, rapid, general and cost-efficient super-resolution imaging method, which can be directly employed in a simple fluorescent imaging system with general fluorophores. Based on diffusion-assisted Förster resonance energy transfer (FRET), fluorescent donor molecules that label specific target structures can be stochastically quenched by diffusing acceptor molecules, thereby temporally separating otherwise spatially overlapped fluorescence signals and allowing super-resolution imaging. The proposed method provides two- to three-fold-enhancement in spatial resolution, a significant optical sectioning property, and favorable temporal resolution in live-cell imaging. We demonstrate super-resolution live-cell dynamic imaging using general fluorophores in a standard epi-fluorescence microscope with light-emitting diode (LED) illumination. Due to the simplicity of this approach, we expect that the proposed method will prove an attractive option for super-resolution imaging.
\end{abstract}

F luorescence imaging of live cells plays a crucial role in the study of biological processes at the cellular and subcellular levels. The diffraction property of light, however, limits the spatial resolution of conventional fluorescence microscopy to $\sim 250 \mathrm{~nm}$ and $\sim 600 \mathrm{~nm}$ in the lateral and axial directions, respectively (Fig. 1a). A series of super-resolution microscopy techniques have been developed to overcome the diffraction limit ${ }^{1,2}$, including techniques based on stimulated emission depletion or patterned illumination in order to confine the fluorescence to a sub-diffraction-sized area or volume [such as stimulated emission depletion (STED) microscopy $^{3,4}$, ground-state depletion (GSD) $)^{5}$, reversible optically linear fluorescence transitions (RESOLFT) ${ }^{6}$, and saturated structured-illumination microscopy $\left.(\mathrm{SSIM})^{7}\right]$ or techniques that are based on the repeated on/off switching of fluorescent probes with single-molecule localization [such as photo-activated localization microscopy (PALM $)^{8,9}$, and stochastic optical reconstruction microscopy (STORM $)^{10,11}$. Recently, a breakthrough in super-resolution imaging techniques based on single-molecule localization has been achieved by using standard fluorescent molecules or dyes ${ }^{12,13}$ : direct STORM (dSTORM) ${ }^{14}$, fluorescence-PALM ${ }^{15}$, bleaching/blinkingassisted localization microscopy (BaLM), ground-state depletion imaging (GSDIM) $)^{16}$, Bayesian analysis of blinking and bleaching $(3 \mathrm{~B} \text { analysis })^{17}$, and imaging membrane structures with lipophilic cyanine dyes ${ }^{18}$.

Although recent super-resolution microscopy techniques can achieve spatial resolutions up to tens of nanometers, these methods still have practical limitations to their direct employment in general biological studies. Most of the existing super-resolution imaging techniques require special imaging systems illuminated with appropriate powers and wavelengths. STED requires high power illumination in order to generate a sharpened excitation spot much smaller than the diffraction-limited focus. The RESOLFT and SSIM methods also generally require the complex alignment of specialized optical system. On the other hand, methods based on singlemolecule localization, such as STORM and PALM, are temporally demanding. They generally require photoswitchable probes to activate and excite the probes with a sufficient number of photons for highly accurate localization. They also require a large number of measurements proportional to the number of fluorophores. In addition, they require high signal-to-noise ratios. This prevents the use of conventional epi-fluorescence 


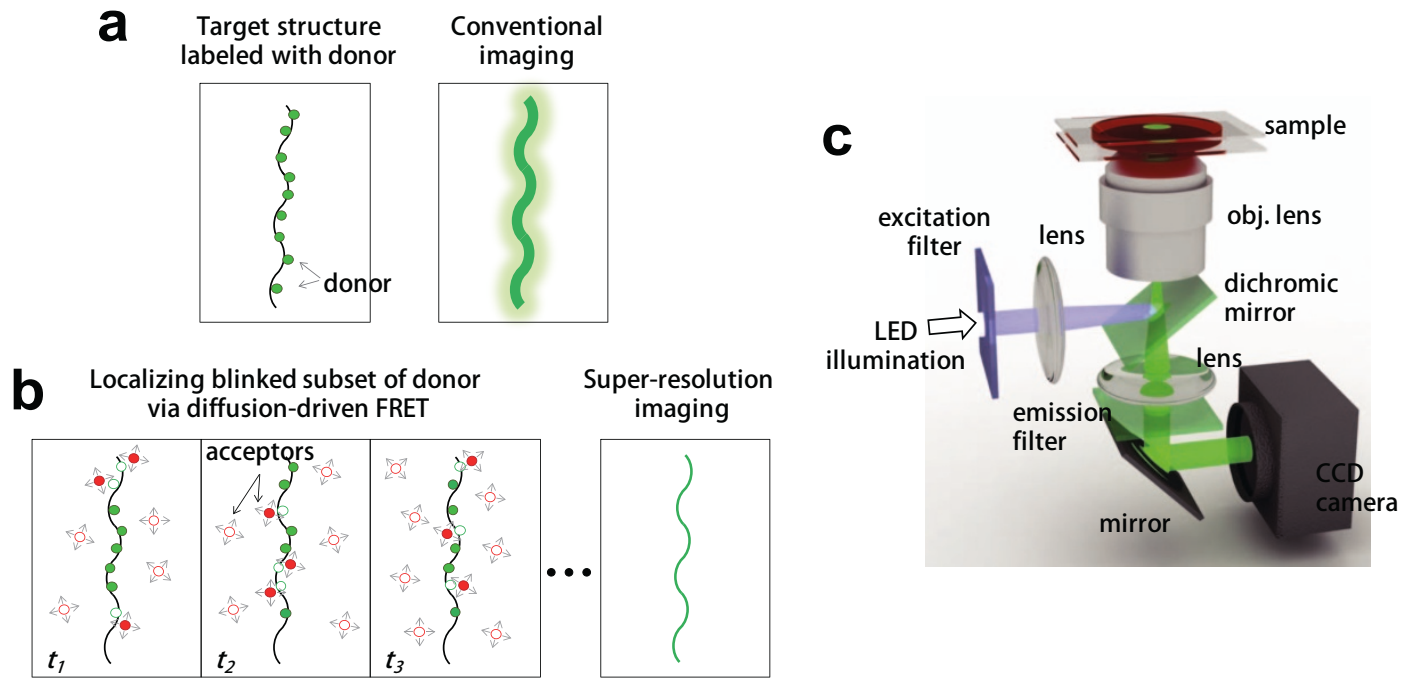

Figure $1 \mid$ Principle of dSOFI. (a-b) Schematic representation of (a) conventional fluorescence imaging and (b) dSOFI principle. (c) Experimental set up of dSOFI imaging.

microscopy but limits the total internal reflection (TIRF) excitation geometry. In addition, labeling intracellular structures with efficient reversible photo-switching fluorescence still remains challenging.

Recently, a statistical analysis method, super-resolution optical fluctuation imaging $(\mathrm{SOFI})^{19}$, was introduced. Without requiring specialized equipment, SOFI utilizes a correlation of temporal fluctuations between neighboring pixels, which can provide a superresolution image with a high signal-to-noise ratio ${ }^{20}$ by calculating the cumulant ${ }^{19,21}$. The enhancement of spatial resolution depends on the order of analysis; calculating the $n^{\text {th }}$ order cumulant provides a factor of $n$ improvement ${ }^{21}$. SOFI has shown its potential to be employed in general optical imaging systems as long as fluorescent signals are randomly fluctuating in time. However, until very recently, SOFI had been only applicable for samples with immunelabeled quantum dot and organic dyes that have intrinsic blinking characteristics ${ }^{19,22,23}$. Recently, photochromic stochastic optical fluctuation imaging ( $\mathrm{pcSOFI}$ ) has been introduced, which utilizes singlemolecule fluctuation using reversibly photochromic labels ${ }^{24}$. Illuminating a reversibly photo-switching fluorescent protein at specific wavelengths, genetically encodable labels can be used for SOFI. However, pcSOFI still face limitations since it requires special reversibly photochromic labels as well as lasers with appropriate wavelengths; these complications still hinder direct applications by many potential users.

Here, we report a simple but powerful technique for super-resolution fluorescence imaging with diffusion-assisted Förster resonance energy transfer (FRET). Fluorescent donor molecules that label target structures can be stochastically quenched in the presence of diffusing acceptor molecules, resulting in the temporal separation of otherwise spatially overlapped fluorescence signals and allowing super-resolution imaging. The proposed technique does not rely on either photo-bleaching events of fluorophores or complex image analysis; thus, it can be readily employed in existing imaging systems.

Since our approach uses general fluoresphores including conventional dyes and typical fluorescent proteins in a conventional eqi-fluorescence microscopy with general illumination - even with light-emitting device (LED) illumination, we refer to it as "direct" SOFI, dSOFI. Our method is based on SOFI reconstruction, and key advantages of SOFI have been shared, namely, its technical simplicity, a broad range of imaging conditions, and a simple imaging process only requiring consecutive images and applying SOFI analysis. The dSOFI method using general fluorophores in a standard epi-fluorescence microscope improves spatial resolution by a factor of two with a markedly improved signal-to-noise ratio and improved optical sectioning capability. We demonstrate the potential of dSOFI under a broad range of imaging conditions, including labeling antibodies, chemically tagged fluorescent dyes, and genetically encoded proteins in dynamic live-cell imaging. We present the sub-diffraction resolution fluorescence imaging of in vitro microtubules, actin, and intermediate filaments of live $\mathrm{NIH} 3 \mathrm{~T} 3$ cells, as well as their dynamics.

\section{Results}

dSOFT principle. The dSOFI method uses general (instead of intrinsically blinking) fluorophores in a conventional epi-fluorescent microscope system. FRET is energy transfer from a donor to an acceptor molecule, with the FRET efficiency depending on the sixth power of the distance between the two ${ }^{25}$. Usually, $R_{0}$ (the distance at which the FRET efficiency is $50 \%$ ) is approximately 5$10 \mathrm{~nm}$. FRET has been widely used to study single molecular interaction by precise measurement of the distance between two molecules ${ }^{26}$. In dSOFI, we exploit FRET and the diffusion process to induce the stochastic blinking of general fluorophores, the combination of which results in super-resolution fluorescence imaging. Generally, fluorophores do not exhibit significant blinking fluorescence, but the addition of freely diffusive acceptor molecules, which appropriately form a FRET pair with fluorophores, makes the target labeling fluorophore molecules repeatedly blink with fluorescence. Like conventional fluorescence imaging, fluorophores as donor molecules are labeled on the target structures.

In dSOFI, FRET acceptor molecules are introduced and they diffuse freely. FRET occurs only when a diffusive acceptor molecule approaches a donor molecule closely enough and the donor fluorophore transiently stops exhibiting fluorescence. After the acceptor molecule move away, the donor fluorophore resumes exhibiting fluorescence. Thus, this short range $(5-10 \mathrm{~nm})$ and random temporal FRET causes uncorrelated fluorescence fluctuation on nearby donor fluorophores. Through the analysis of this uncorrelated fluorescence fluctuation signal, a super-resolution image of target molecules can be achieved (Fig. 1b).

In vitro microtubule imaging. To demonstrate the principle of dSOFI and compare its performance with that of conventional microscopy, we performed in vitro measurement of microtubules stained with fluorescent amino-methyl-coumarin-acetate (AMCA) dye, which had $350 / 450 \mathrm{~nm}$ excitation/emission wavelengths. 

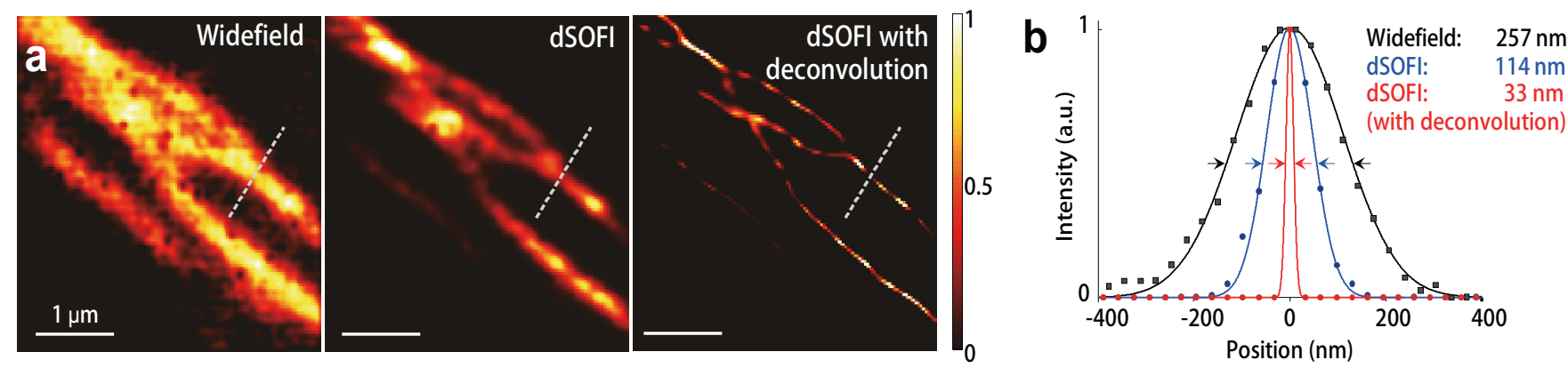

Figure $2 \mid$ dSOFI in vitro images. (a) in vitro images of microtubules measured with fluorescence wide-field microscopy (left), dSOFI (middle), and dSOFI with deconvolution (right). Microtubules are labeled with AMCAs that were stochastically quenched with diffusive PYPs. (b) Cross-sectional profiles along the dashed lines in (a) with FWHM values.

Photoactive yellow protein (PYP) was used as an acceptor molecule to form a FRET pair with AMCA. A series of fluorescence microscopic images were measured with a conventional epi-fluorescence microscope (Fig. 1c) and then analyzed with the SOFI algorithm ${ }^{19}$. The reconstructed dSOFI image is shown in Fig. 2a. The dSOFI method improved the resolution two-fold compared with that of a conventional wide-field fluorescent image. After applying a deconvolution algorithm to the DSOFI image, this method achieved a spatial resolution down to a full-width half-maximum (FWHM) of $32.5 \mathrm{~nm}$ (Fig. 2b), which is an almost eight-fold improvement compared to the performance of diffraction-limited conventional fluorescence microscopy. The dSOFI images obtained in this study clearly reveal individual microtubule structures, which are not resolved in the conventional wide-field fluorescence image.

Stochastic quenching by diffusing acceptor molecules. To demonstrate that the resolution enhancement in dSOFI primarily results from FRET-induced fluorescence blinking and not from the photo-bleaching effect, we analyzed temporal fluorescence signals from sequential raw fluorescence images. If fluorescence molecules labeled at target structures blink enough to localize the target structures within the diffraction spot size, the temporal fluorescence signals from the structure must be differentiable from signals from nearby points, which can be quantified by calculating correlation or covariance. We analyzed the fluorescence signals of a single microtubule fiber labeled with AMCA (Fig. 3a) in the absence and (Fig. 3b) in the presence of corresponding FRET molecule PYPs, respectively. Both cases show comparable spatial resolution since no SIFT analysis was performed in Figure 3b. Then, we calculate the cross-correlation between various spatial positions:

$$
\langle I * I\rangle\langle\Delta r\rangle=\int I(r, \tau) I(r+\Delta r, t+\tau) d \tau
$$

The normalized cross temporal correlations between pixels on the white dashed lines denoted in Figs. $3 a-b$ are shown in Figs. $3 c-d$, respectively. The widths of cross-correlation normal to the main diagonal line show how much temporal fluorescence signals are correlated; only uncorrelated temporal signals can be distinguished by SOFI reconstruction. Thus, the widths of cross-correlation normal to the main diagonal line correspond to the spatial resolving power.

In the absence of appropriate FRET acceptor pairs, the target molecules did not exhibit significant blinking fluorescence, and fluorescence signals on the microtubule structure showed strong spatial correlation between nearby pixels along off-diagonal directions with the diffraction spot size $(250 \mathrm{~nm} \sim 5$ pixels, Fig. $3 \mathrm{c})$. This indicates a strong correlation between pixels within a diffraction spot size; thus, there is no significant resolution enhancement. However, in the presence of FRET acceptor molecules, fluorescence signals on the microtubule structure presented little spatial correlation,
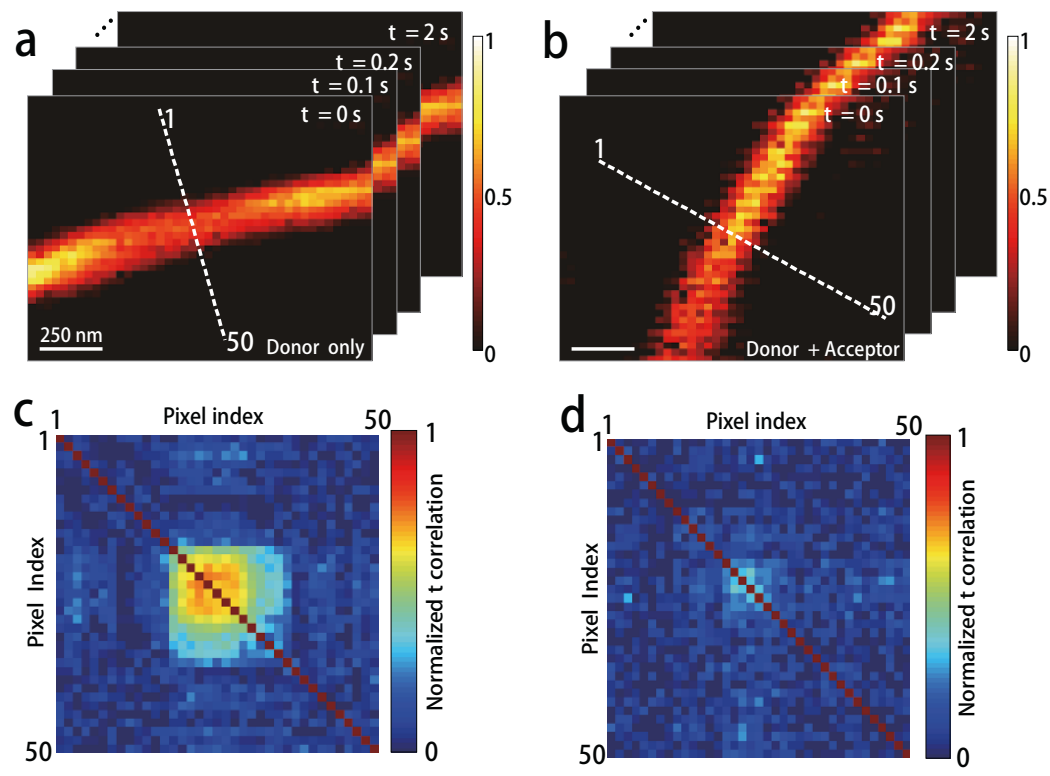

Figure 3 Temporal signal analysis in dSOFI images. (a-b) Consecutively acquired fluorescent images of a microtubule fiber in (a) the absence and (b) presence of FRET acceptor molecules. (c-d) Corresponding temporal correlation of fluorescence signal. 
indicating that blinking caused by stochastic FRET events can improve spatial resolving power when combined with SOFI analysis (Fig. 3d).

Acceptor concentration on dSOFI analysis. To study the effects of acceptor concentration on dSOFI analysis, we performed in vitro microtubule imaging experiments with various concentrations of PYP. At a fixed donor concentration of fluorescent amino-methylcoumarin-acetate (AMCA) dye which stain microtubules (See Methods), we performed experiments with a diverse range of PYP concentrations $(0 \mu \mathrm{M}, 0.5 \mu \mathrm{M}, 2.5 \mu \mathrm{M}, 5 \mu \mathrm{M}, 7.5 \mu \mathrm{M}, 10 \mu \mathrm{M}$, $12.5 \mu \mathrm{M}, 15 \mu \mathrm{M}, 25 \mu \mathrm{M}$, and $45 \mu \mathrm{M})$. The results show that there is an optimal range of acceptor concentration $(7.5-12.5 \mu \mathrm{M})$ in which dSOFI analysis effectively works with good repeatability. Too high or too low acceptor concentration easily frustrates the blinking of donor molecules to the extent that SOFI reconstruction does not work effectively. Under a low concentration regime, most donor molecules will emit fluorescence; however, a few FRET events will occur at single-molecule levels. On the other hand, with a high concentration of acceptor molecules, most donor molecules will exhibit ensemble FRET events; thus, induced blinking is not sufficient for dSOFI analysis. At an optimal concentration of acceptor molecules, diffusion-assisted FRET will cause appropriate small ensemble of blinking ${ }^{27}$, which can be used for SOFI reconstruction.

To further demonstrate the principle of dSOFI with control groups, we measured polystyrene beads with a diameter of $10 \mu \mathrm{m}$ coated with AMCA dye (See Methods) under various imaging conditions. First, conventional wide-field images of the beads show diffraction-limited spatial resolution irrespective of the presence or absence of acceptor molecules, PYP (Fig. 4a). When SOFI reconstruction is applied to the images of the beads in the presence of PYP, it produces super-resolution images of the beads. However, in the absence of PYP, SOFI reconstruction does noting, and it still gives diffraction-limited images.

Optical sectioning in dSOFI. To evaluate the optical sectioning capability of dSOFI, we measured the depth-of-field by imaging polystyrene beads with a diameter of $10 \mu \mathrm{m}$ coated with AMCA dye. The wide-field fluorescence images of the beads with and without PYP protein as an acceptor molecule are diffractionlimited with large oscillatory patterns caused by optical diffraction (Fig. 4a). When the dSOFI method was applied, the edge of the bead was visualized with a very shape profile. This result demonstrates the optical sectioning capability of dSOFI as well as enhanced spatial resolution it achieves. To quantitatively analyze the depth-of-field, the intensity profiles measured by wide-field imaging and dSOFI are shown as functions of the lateral position (Fig. 4b). In the wide-field image, strong intensity signals were seen inside the radius of the bead; fluorescent dyes at defocus planes were seen due to long depth-offield. However, the dSOFI image shows a very sharp profile at the edge, which demonstrates that the depth-of-field is significantly reduced. The axial positions of the bead corresponding to the position with $50 \%$ fluorescent intensity are $2.2 \mu \mathrm{m}$ and $1 \mu \mathrm{m}$ for the wide-field image and dSOFI image, respectively.

Super-resolution live-cell imaging of F-actin and $\alpha$-tubulins. To demonstrate the capability of dSOFI in super-resolution live-cell imaging, we visualized two major cytoskeleton structures in live cells (see Methods section). Filamentous actin (F-actin) and $\alpha$ tubulins in NIH 3T3 cells were visualized with Lifeact (F-actin marker) and $\alpha$-tubulin labeled with cerulean [a cyan fluorescence protein (CFP) variant]. Citrine [a yellow fluorescence protein (YFP) variant] is expressed in the cytoplasm as a FRET pair to cerulean. Cerulean and citrine are a FRET pair widely used in live cells. Using dSOFI, F-actin (Fig. 5a) and microtubule structures (Fig. 5b) in live $\mathrm{NIH} 3 \mathrm{~T} 3$ cells were visualized. While conventional fluorescence microscopy only provides diffraction-limited blurry images of the
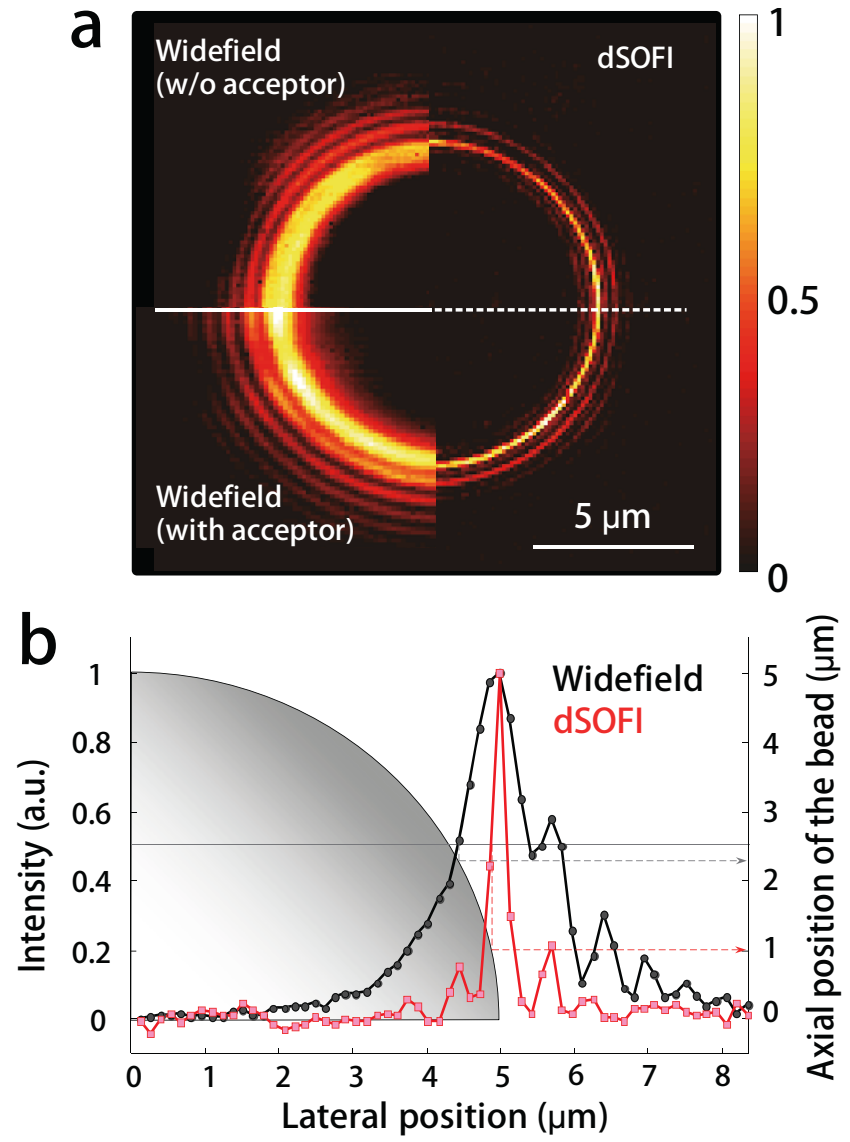

Figure $4 \mid$ Optical sectioning capability of dSOFI. (a) images of polystyrene beads coated with fluorescent protein measured with fluorescence wide-field microscopy in the absence of acceptor (top left), in the presence of acceptor (bottom lef), and dSOFI (right). (b) Crosssectional profiles along the dashed lines in (a).

structures, the dSOFI images reveal the high structural details of Factin and the microtubule structures at a resolution of $\sim 100 \mathrm{~nm}$ (box i-vi in Figs. 5a-b and cross-sectional plot in Fig. 5c).

Note that acceptor molecules can also be introduced into cell cytoplasm via various alternative routes, including micro-injection, electroporation, endocytosis, and so on. However, here we employed a double-expression method to achieve the physiologically relevant concentration of both acceptor and donor molecules. Both the cerulean and citrine were genetically expressed in vivo, and super-resolution imaging has been effectively demonstrated, suggesting that dSOFI still works for the physiological conditions of live cells.

More importantly, dSOFI allows us to visualize the dynamics of living cells over a long period and at extremely high spatial resolving power, since dSOFI techniques do not require the fixation of biological samples. F-actin structures labeled with cerulean in a live $\mathrm{NIH}$ 3 T3 cell were imaged with dSOFI every 5 min during a period of $15 \mathrm{~min}$ (Fig. 6). The dynamics of actin polymerization and cytoskeleton movements were captured in extremely specific detail (green and blue arrows in Fig. 6). Since the analysis of blinking fluorescence signal with the SOFI algorithm provides optical sectioning capability as well as enhanced resolving power ${ }^{19}$, the dynamics of F-actin fluctuation in the axial direction can be observed.

\section{Discussion}

In conclusion, we present a novel super-resolution microscopic technique, dSOFI, which is easily transferable to conventional fluorescent microscope imaging systems. The dSOFI method represents the first 

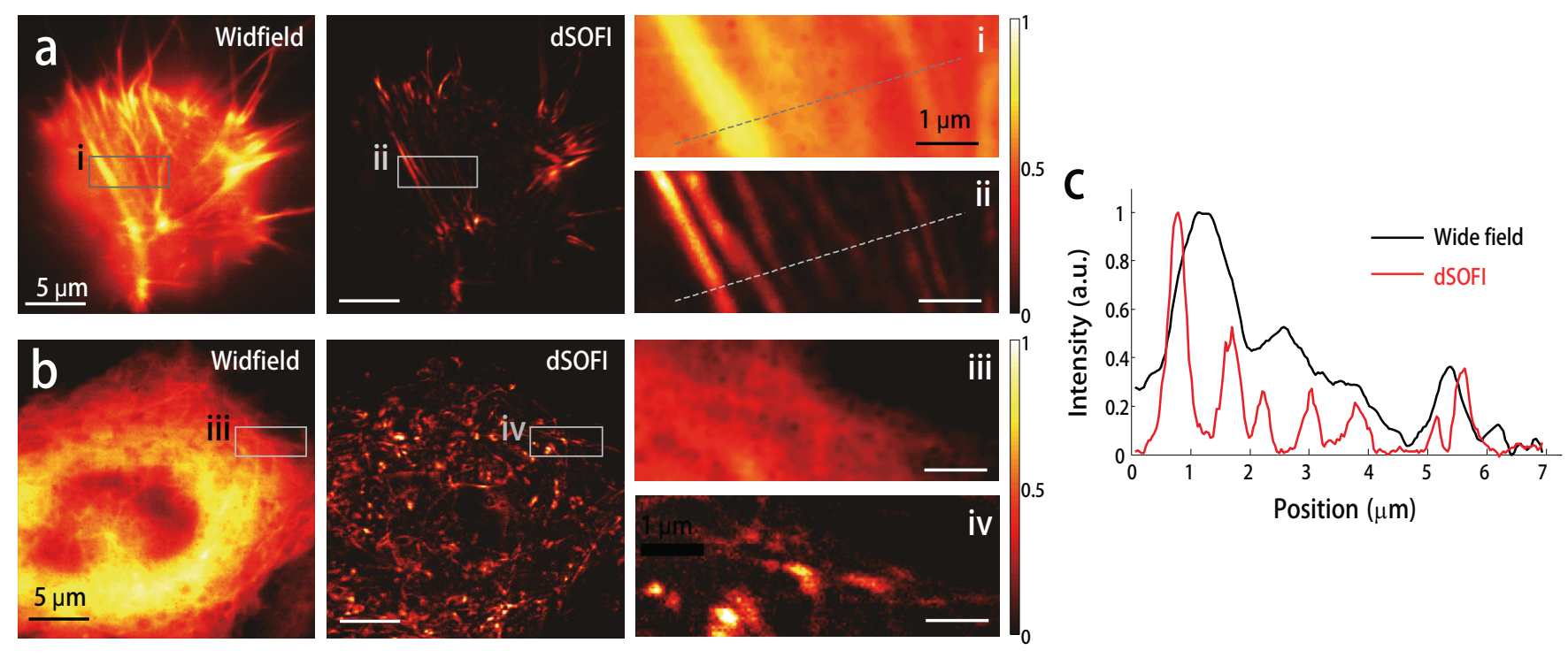

Figure 5 | Live-cell dynamic imaging using dSOFI. (a) Actin-ceruline expressed in live NIH 3T3 cell imaged with conventional wide-field fluorescence microscopy (left) and dSOFI (middle). Cytoplasmic citrine acts as a FRET acceptor. (i, ii) Magnifications of the white box regions. (b) Tubulincerulean expressed in live NIH 3T3 cell imaged with conventional wide-field fluorescent microscopy (left) and dSOFI (middle). (iii, iv) Magnifications of the white box regions.

attempt to achieve super-resolution imaging in live cells using FRET $^{28}$; dSOFI exploits diffusion-assisted FRET to achieve stochastically fluctuating florescence from standard fluorophores - fluorescent proteins and dyes. This new technique achieves dynamical super-resolution imaging in live cells. dSOFI is a very simple, general and cost-efficient super-resolution imaging method, which can be used directly in existing fluorescent imaging systems with general fluorophores. Using this method, we demonstrated super-resolution live-cell imaging with standard non-photoswitchable fluorophores under a conventional epi-fluorescent microscope equipped with LED illumination. Simply by adding diffusive acceptor molecules near target structures, anyone can produce super-resolution molecular imaging with existing instrumentation.

In addition, the dSOFI technique provides extremely fast imaging acquisition and analysis, especially compared to techniques based on the localization of individual fluorescent molecules ${ }^{10,29}$. Only 300 raw fluorescent image measurements, typically captured at video frame rate of $10-50 \mathrm{~Hz}$, are required to reconstruct one super-resolution dSOFI image as shown in Fig. 1d. Considering the typical diffusion properties of fluorescent molecules, comparable resolution improvement is expected to be achieved up to an acquisition speed of $0.1 \mathrm{~ms}$ using the proposed method. Depending on the acquisition speed of the camera, the fast dynamics of biological systems can be visualized at super-resolution. In addition, to analyze a region of around $25 \times$ $25 \mu \mathrm{m}$, processing takes $\sim 3.5 \mathrm{~s}$ on a typical desktop computer (Intel Core i5-2430M CPU, $2.40 \mathrm{GHz}$ ).

As demonstrated here for a fluorescent dye - protein pair and a florescent protein - protein pair, the principle of dSOFI should work for any FRET pairing molecules. Conventional fluorescent microscope imaging systems can easily adopt this system. The simple addition of an acceptor that can form a FRET pair with the target labeling fluorophore, and the analysis of the blinking fluorescence
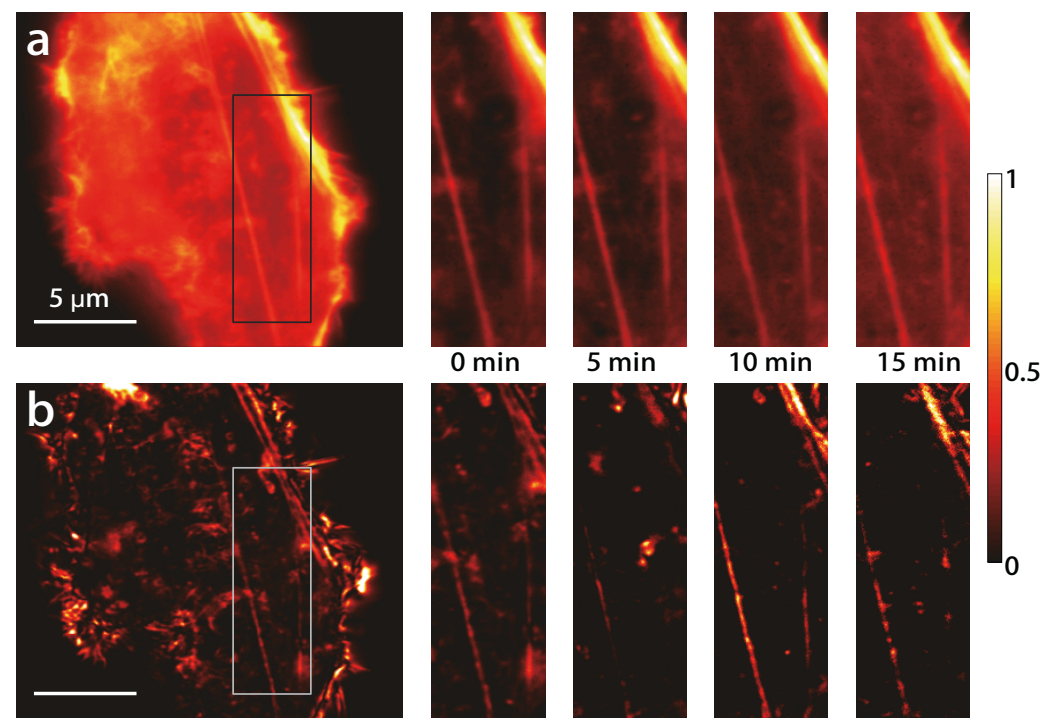

Figure 6 | Long-time duration dynamic images of actin-ceruline expressed live NIH 3T3 cells. (a) imaged with wide-field fluorescence microscopy. (b) imaged with dSOFI. Magnified images of the box regions measured every 5 min up to 15 min. 
signal can produce a super-resolution image. No special photo-activatable proteins or complex optical systems are required. As demonstrated in this study, even LED illumination can be used since the proposed technique does not require high-intensity illumination. However, the proposed method also bears some practical limitations. For example, the use of a FRET pair to obtain a single-channel image may complicate sample preparation and make multi-color imaging difficult. Nonetheless, the proposed approach still has appealing advantages, namely, the simplicity of a method that only requires a FRET pair to induce the blinking of labeling molecules as well as the fact that potential users can utilize existing imaging systems.

In summary, we demonstrated the viability of this principle through the imaging of cytoskeleton structures in vitro and as expressed in live cells. Our new technique is sufficiently broad and general to allow for potential application in cell biology, biophysics, biochemistry, biotechnology, and biomedicine, wherever super-resolution molecular imaging can have an impact. All users of microscopes can use their own super-resolution fluorescence microscopes to apply the present technique. Thus, dSOFI will immediately find direct and versatile applications in diverse fields such as cell biology, biophysics, and biochemistry; therefore, we believe that our new method represents a vast improvement on existing super-resolution imaging technologies, especially in live-cell studies.

\section{Methods}

Microtubule preparation. AMCA-labeled microtubules were assembled at $35^{\circ} \mathrm{C}$ for $20 \mathrm{~min}$ from $0.91 \mu \mathrm{M}(0.1 \mathrm{mg} / \mathrm{ml})$ tubulin (from Cytoskeleton, cat. \# TL440M) in $\mathrm{PEM}_{50}$ buffer (50 mM Pipes, $1 \mathrm{mM}$ EGTA, $1 \mathrm{mM} \mathrm{MgCl}_{2}, \mathrm{pH} 6.8$ ) in the presence of $1 \mathrm{mM}$ GTP and $5 \mathrm{wt} \%$ glycerol and then stabilized by $0.91 \mu \mathrm{M}$ taxol $(2 \mathrm{mM}$ taxol in DMSO was added in a stepwise manner $)^{30}$. Purified PYP was synthesized based on Ref. 31. PYP was concentrated to $5 \mu \mathrm{M}$ with PEM50 buffer. We mixed the microtubule solution and $5 \mu \mathrm{M}$ PYP solution in $1: 1$ volume ratio.

Fluorescence bead preparation. $50 \mu \mathrm{l}$ of aliphatic amine latex beads with a diameter of $4.5 \mu \mathrm{m}$ (Invitrogen, Molecular probes, Inc. A37370) from the stock solution $(2 \times$ $10^{10}$ beads $/ \mathrm{ml}$ ) was diluted to a volume of $1 \mathrm{ml}$ in distilled water. The bead solution was centrifuged at $3600 \mathrm{rpm}$ for 15 minutes and re-suspended in a $90 \mu \mathrm{PBS}(\mathrm{pH}$ 7.4). $50 \mu \mathrm{g}$ of Alexa350 (Invitrogen, Alexa Fluor ${ }^{\circledR} 350$ NHS esters, A10168) NHS esters and $10 \mu \mathrm{l}$ of sodium bicarbonate are then added to the bead solution and incubated at room temperature in a tube rotator for 30 minutes. Then, Alexa350/bead solution was subjected to three centrifugations ( $3600 \mathrm{rpm}$ for 15 minutes) and resuspension steps (PBS, $\mathrm{pH} 7.4$ ) to remove any free Alexa350 NHS esters.

Cell preparation: cell culture and transfection. NIH 3T3 cells were purchased from American Type Culture Collection (ATCC). Cells were cultured in Dulbecco's modified Eagle's medium (DMEM, PAA) supplemented with $10 \%$ fetal bovine serum (FBS, Gibco) and $1 \%$ antibiotics - anti mycotics. NIH 3T3 cells were maintained in $10 \% \mathrm{CO} 2$ at $37^{\circ} \mathrm{C}$. NIH $3 \mathrm{~T} 3$ cells were plated on glass bottom 96-well plates (Metrical Inc.) and plated on poly-D-lysine-coated glass bottom 96-well plates. Transfection was performed one day after plating, by using lipofectamine 2000 (Invitrogen) with $500 \mathrm{ng}$ of DNA according to manufacturer's protocol. Transfected cells were incubated about $24 \mathrm{hrs}$ before imaging.

Cell preparation: DNA constructs. Cerulean-Tubulin constructs were made by subcloning tubulin, Human tubulin alpha 1B, from the pAcGFP-Tubulin (Clontech) plasmids into Cerulean- $\mathrm{Cl}^{32}$ using XhoI and BamHI restriction enzymes. Lifeact fragment were generated by annealing method with following primers: sense $5^{\prime}$ AATTCTGGTGTCGCAGATTTGATCAAGAAATTCGAAAGCATCTCAAAGGAAGAAG- ${ }^{\prime}$, antisense $5^{\prime}$-GATCTTCTTCCTTTGAGATGCTTTCGAATTTCTTGATCAAATCTGCGACACCAG- $3^{\prime 33}$. Lifeact fragment was cloned into Cerulean-C1 using EcoRI and BamHI restriction enzymes. Citrine-C1 plasmids that were used in this study were previously described ${ }^{32}$.

Imaging system and acquisition. A conventional optical microscope system (IX-71, Olympus) equipped with an objective lens (UIS2 $100 \times$, Olympus) was used for SIFT. An UV LED (M385L2-C1, Thorlabs, 270-430 $\mathrm{mW}$ ) or a white-light LED (MCWHL2-C1, Thorlabs, 650-700 $\mathrm{mW}$ ) was used to excite the donor molecules that labeled the target samples. For the AMCA-PYP FRET pair, the UV LED was used for excitation. A dichroic mirror (MD416, Thorlabs) and an emission filter (FB460-10, Thorlabs) mounted in a fluorescent filter cube (IX-RFA, Olympus) were used to acquire fluorescence image. For the Cerulean-Citrine FRET pair, the white-light LED (MCWHL2-C1, Thorlabs) source was used for illumination. An excitation filter (FF01-438/24, Semrock), an emission filter (FF01-483/32, Semrock), and a dichroic mirror (FF458-Di01, Semrock) were used to acquire fluorescence image. The illumination powers at the sample plane for AMCA and CFP are $9 \mathrm{~W} / \mathrm{cm}^{2}$ and $1.3 \mathrm{~W} /$ $\mathrm{cm}^{2}$, respectively. For an imaging system, we employed a high magnification objective lens (UIS2 $100 \times$, Olympus) with 1.30 NA and a sCMOS camera (NEO, Andor) with a $6.5 \mu \mathrm{m}$ pixel size. The camera was directly adapted to a $1 \times$ magnification port of IX71 , and one pixel size of the detector corresponds to $65 \mathrm{~nm}$ at the sample plane. We typically acquired 300-1000 consecutives images with 10-50 frames per second.

Data processing. To retrieve a super resolution image from the measured fluorescent images, we analyze the data with a SOFIT algorithm using a custom-made code in MatLab software (Mathworks Inc., Natick, MA). Before analysis, raw images were digitally magnified by factor of 2 . We calculated the $2^{\text {nd }}$ or $4^{\text {th }}$-order auto-cumulant SOFI ( $2^{\text {nd }}$ or $\left.4^{\text {th }} \mathrm{AC}-\mathrm{SOFI}\right)$ by using the shortest accessible lag time (the frame integration time) and then deconvolved the image by using a two-dimensional Gaussian function, whose full-width half-maximum is a size of diffraction limit of the used optical imaging system, enploying deconvlucy (Lucy-Richardson) command ${ }^{34}$.

1. Hell, S. W. Far-field optical nanoscopy. Single Molecule Spectroscopy in Chemistry, Physics and Biology 365-398 (2010).

2. Huang, B., Babcock, H. \& Zhuang, X. Breaking the diffraction barrier: superresolution imaging of cells. Cell 143, 1047-1058 (2010).

3. Hell, S. W. \& Wichmann, J. Breaking the diffraction resolution limit by stimulated emission: stimulated-emission-depletion fluorescence microscopy. Optics letters 19, 780-782 (1994)

4. Klar, T. A., Jakobs, S., Dyba, M., Egner, A. \& Hell, S. W. Fluorescence microscopy with diffraction resolution barrier broken by stimulated emission. Proceedings of the National Academy of Sciences 97, 8206 (2000).

5. Hell, S. W. \& Kroug, M. Ground-state-depletion fluorscence microscopy: A concept for breaking the diffraction resolution limit. Applied Physics B: Lasers and Optics 60, 495-497 (1995).

6. Hofmann, M., Eggeling, C., Jakobs, S. \& Hell, S. W. Breaking the diffraction barrier in fluorescence microscopy at low light intensities by using reversibly photoswitchable proteins. Proceedings of the National Academy of Sciences of the United States of America 102, 17565-17569 (2005).

7. Gustafsson, M. Nonlinear structured-illumination microscopy: Wide-field fluorescence imaging with theoretically unlimited resolution. Proceedings of the National Academy of Sciences of the United States of America 102, 13081 (2005)

8. Betzig, E. et al. Imaging intracellular fluorescent proteins at nanometer resolution. Science 313, 1642 (2006).

9. Hess, S. T., Girirajan, T. P. K. \& Mason, M. D. Ultra-High Resolution Imaging by Fluorescence Photoactivation Localization Microscopy. Biophys J 91, 4258-4272 (2006).

10. Rust, M. J., Bates, M. \& Zhuang, X. Sub-diffraction-limit imaging by stochastic optical reconstruction microscopy (STORM). Nature Methods 3, 793-796 (2006).

11. Bates, M., Huang, B., Dempsey, G. T. \& Zhuang, X. Multicolor super-resolution imaging with photo-switchable fluorescent probes. Science $317,1749-1753$ (2007).

12. Vogelsang, J. et al. Make them Blink: Probes for Super-Resolution Microscopy. ChemPhysChem 11, 2475-2490 (2010).

13. van de Linde, S., Heilemann, M. \& Sauer, M. Live-Cell Super-Resolution Imaging with Synthetic Fluorophores. Annual Review of Physical Chemistry 63, 519-540 (2012).

14. Heilemann, M. et al. Subdiffraction-Resolution Fluorescence Imaging with Conventional Fluorescent Probes. Angewandte Chemie International Edition 47, 6172-6176 (2008).

15. Hess, S. T., Girirajan, T. P. K. \& Mason, M. D. Ultra-high resolution imaging by fluorescence photoactivation localization microscopy. Biophys J 91, 4258 (2006)

16. Fölling, J. et al. Fluorescence nanoscopy by ground-state depletion and singlemolecule return. Nature Methods 5, 943-945 (2008).

17. Burnette, D. T., Sengupta, P., Dai, Y. H., Lippincott-Schwartz, J. \& Kachar, B. Bleaching/blinking assisted localization microscopy for superresolution imaging using standard fluorescent molecules. Proceedings of the National Academy of Sciences of the United States of America 108, 21081-21086 (2011).

18. Shim, S. H. et al. Super-resolution fluorescence imaging of organelles in live cells with photoswitchable membrane probes. Proceedings of the National Academy of Sciences 109, 13978-13983 (2012).

19. Dertinger, T., Colyer, R., Iyer, G., Weiss, S. \& Enderlein, J. Fast, background-free, $3 \mathrm{D}$ super-resolution optical fluctuation imaging (SOFI). Proceedings of the National Academy of Sciences 106, 22287 (2009).

20. Geissbuehler, S., Dellagiacoma, C. \& Lasser, T. Comparison between SOFI and STORM. Biomedical optics express 2, 408-420 (2011).

21. Dertinger, T., Colyer, R., Vogel, R., Enderlein, J. \& Weiss, S. Achieving increased resolution and more pixels with Superresolution Optical Fluctuation Imaging (SOFI). Optics Express 18, 18875-18885 (2010).

22. Dertinger, T., Heilemann, M., Vogel, R., Sauer, M. \& Weiss, S. Superresolution optical fluctuation imaging with organic dyes. Angewandte Chemie 122, 9631-9633 (2010).

23. Hoyer, P., Staudt, T., Engelhardt, J. \& Hell, S. W. Quantum Dot Blueing and Blinking Enables Fluorescence Nanoscopy. Nano letters (2011).

24. Dedecker, P., Mo, G. C. H., Dertinger, T. \& Zhang, J. Widely accessible method for superresolution fluorescence imaging of living systems. Proceedings of the National Academy of Sciences 109, 10909-10914 (2012).

25. Förster, T. Delocalized excitation and excitation transfer (Florida State University, 1965). 
26. Ha, T. Single-molecule fluorescence resonance energy transfer. Methods 25, 78-86 (2001).

27. Mukhopadhyay, S. \& Deniz, A. A. Fluorescence from diffusing single molecules illuminates biomolecular structure and dynamics. Journal of fluorescence 17 , 775-783 (2007).

28. Grecco, H. E. \& Verveer, P. J. FRET in Cell Biology: Still Shining in the Age of Super-Resolution? ChemPhysChem 12, 484-490 (2011).

29. Cox, S. et al. Bayesian localization microscopy reveals nanoscale podosome dynamics. Nat Meth 9, 195-200 (2012).

30. Choi, M. et al. Human microtubule-associated-protein tau regulates the number of protofilaments in microtubules: a synchrotron x-ray scattering study. Biophys $J$ 97, 519-527 (2009).

31. Imamoto, Y., Ito, T., Kataoka, M. \& Tokunaga, F. Reconstitution photoactive yellow protein from apoprotein and p-coumaric acid derivatives. FEBS letters 374 , 157-160 (1995).

32. Rizzo, M. A., Springer, G. H., Granada, B. \& Piston, D. W. An improved cyan fluorescent protein variant useful for FRET. Nature biotechnology 22, 445-449 (2004).

33. Riedl, J. et al. Lifeact: a versatile marker to visualize F-actin. Nature methods 5 , 605-607 (2008).

34. Biggs, D. S. C. \& Andrews, M. Acceleration of iterative image restoration algorithms. Applied optics 36, 1766-1775 (1997).

\section{Acknowledgments}

This work was supported by KAIST, KAIST Institute for Optical Science and Technology, HRHRP, MEST/NRF, IBS, World Class University Program, Korea [2009-0087691(BRL), NRF-2012R1A1A1009082, NRF-2012K1A31A1A09055128, NRF-M3C1A1-048860, NRF-2011-355-C00037, NRF-2011-0030923, NRF-2011-0031931, the Research Center Program (CA1201) of IBS (Institute for Basic Science) in Korea, R33-2008-000-10163-0, NRF- 2012R1A1A1011023]. YKP acknowledges support from TJ ChungAm Foundation.

\section{Author contributions}

S.C. and J.J. designed experiments, performed research, analyzed the data and wrote the paper; C.S., H.L., P.G., T.-Y.Y., M.W.K. and M.C.C. performed research and analyzed the data; H.I., W.D.H. and Y.P. designed experiments, analyzed the data, and wrote the paper.

\section{Additional information}

Competing financial interests: The authors declare no competing financial interests.

License: This work is licensed under a Creative Commons

Attribution-NonCommercial-NoDerivs 3.0 Unported License. To view a copy of this license, visit http://creativecommons.org/licenses/by-nc-nd/3.0/

How to cite this article: Cho, S. et al. Simple super-resolution live-cell imaging based on diffusion-assisted Förster resonance energy transfer. Sci. Rep. 3, 1208; DOI:10.1038/ srep01208 (2013). 